\title{
Pancreatic function in amyotrophic lateral sclerosis ${ }^{1}$
}

\author{
ROBERT A. UTTERBACK, ALVIN J. CUMMINS, CHARLES A. CAPE, \\ AND JULIO GOLDENBERG
}

From the Department of Neurology, University of Tennessee, Memphis, Tennessee, U.S.A.

SUMMARY No abnormality of exocrine function of the pancreas was found in 10 patients with amyotrophic lateral sclerosis. Eight patients showed abnormal glucose metabolism, attributed to the effects of age, malnutrition, diminished physical activity, and decreased muscle mass.

A vague relationship between amyotrophic lateral sclerosis (ALS) and the pancreas has been suspected for years. Barris (1953) reported that occasionally pancreatic adenomas and ALS were associated, and resection of the adenoma could be followed by a remission of the ALS. Ask-Upmark (1950) has described the occurrence of ALS after gastric resection. He suggested that malnutrition may be of some importance. This relationship must be extremely rare because Stiel (1967) did not find any patients with gastric resections in a series of 125 ALS patients. In addition, he found normal gastric secretion in five patients. Abnormalities of glucose utilization have been described (Steinke and Tyler, 1964; Ionăşescu and Luca, 1964) but abnormalities in the exocrine function of the pancreas in ALS were not described until 1967 when Quick and Greer reported a significant number of abnormalities in five patients with ALS. Their findings have recently been challenged (Brown and Kater, 1969). The present clinical study confirms the abnormal glucose utilization, but fails to show any abnormalities in exocrine pancreatic function in 10 patients with ALS. We performed a more complete battery of pancreatic tests than have previously been reported.

\section{CLINICAL MATERIAL}

Six females and four males with ALS and one man with chronic pancreatitis were studied. The diagnosis of ALS was established by the history, physical examination, and clinical course. Necropsies confirmed the diagnosis in three patients with ALS.

\footnotetext{
1This investigation was supported by Public Health Service Research Grant No. RR00211 from the University of Tennessee Clinical Research Center.
}

Electromyography, nerve conductions and cervical spine radiographs were performed in all patients. A complete myelogram was done in selected patients to exclude cervical spondylosis and cervical cord tumours. Encephalitis and poliomyelitis were excluded by the history. Luetic amyotrophy was excluded by the history, non-reactive serologies, and normal cerebrospinal fluid. There was no clinical evidence of significant gastrointestinal symptoms, neoplasia, thyrotoxicosis, or heavy metal poisoning, but extensive testing was performed to rule out these disorders if there were any suggestive symptoms. None of the patients had had gastrectomies. Nine of the ALS patients had a combination of progressive upper and lower motor neurone disease characterized by hyperreflexia, pathological reflexes, wasting, weakness, and fasciculations. Seven of the patients had bulbar muscle weakness manifested by dysphagia, dysarthria, and wasting and weakness of the tongue. Despite the dysphagia only two of our patients were malnourished. The tenth ALS patient had only wasting, weakness, and fasciculations of the arms. There was no evidence of corticospinal tract involvement. Electromyography showed evidence of anterior horn cell damage only in the arms; whereas in the other nine ALS patients there was involvement of both the arms and the legs. A cervical myelogram was done in this tenth case to exclude focal cervical spinal cord pathology. Subsequent out-patient examinations showed the entire clinical picture of amyotrophic lateral sclerosis. The man with chronic pancreatitis had a moderately severe peripheral neuropathy. Chronic alcoholism was thought to be the aetiology of both. Neurological examination and electromyography did not show any evidence of either upper motor neurone disease or anterior horn cell damage. 
METHODS

Conventional methods were used for assay of amylase (Rice, 1959), lipase (Henry, Sobel, and Berkman, 1957) and leucine aminopeptidase (Goldbang and Rutenburg, 1958). Secretin stimulation (Beierwaltes, Johnson, and Solari, 1957) and faecal fat determinations (van de Kamer, 1949) were performed by the gastrointestinal laboratory. The $I^{131}$ triolein test was done as follows: a capsule containing 25 to $50 \mu \mathrm{c}$ was given orally. Blood samples were taken at two, three, four, five, and six hours and the sample with the greatest radioactivity was used to determine the percentage of absorption. The assumed blood volume was calculated by multiplying $7 \cdot 2 \%$ by the patient's weight in kilograms. Normal values were $7 \%$ or more. Intravenous tolbutamide tests were performed by giving $1 \mathrm{~g}$ sodium tolbutamide intravenously over three minutes. Blood samples were taken fasting, and at $20,30,60$, and 120 minutes. Oral glucose tolerance tests were performed on all patients using $75 \mathrm{~g}$ glucola. Abnormal values were greater than $110 \mathrm{mg} / 100 \mathrm{ml}$. fasting, 160 at one hour, 140 at one and a half hours, and 120 at two hours.

\section{RESULTS}

The tests that are appropriate to this study are listed in the Table. The glucose tolerance and tolbutamide tests showed the only consistent abnormalities (Figs. 1 and 2). Three patients with diabetic glucose tolerance curves had tolbutamide tests that confirmed diabetes. Three patients with diabetic glucose tolerance tests had inconclusive tolbutamide tests. Two patients with diabetic curves did not have tolbutamide tests. Finally the two patients with normal glucose metabolism had normal tolbutamide tests. Only one of the two malnourished patients had an abnormal glucose tolerance curve. The tests of exocrine function were all normal.

The abnormalities on the upper gastrointestinal radiograph series consisted of varices and a traction diverticulum of the oesophagus in one patient. Another had a small sliding hernia, and the third had a 'possible duodenal ulcer'. The small bowel abnormalities consisted of some segmentation and coarsening of the small bowel in one patient and a rapid tra ssit time in the other one.

\section{DISCUSSION}

'The glucose tolerance and the tolbutamide tests, both gauges of the endocrine function of the pancreas, were abnormal, whereas the exocrine function was completely normal. Endocrine dysfunction has been observed previously (Steinke and Tyler, 1964; Ionăşescu and Luca, 1964; Quick and Greer, 1967), but Brown and Kater (1969) have refuted these abnormalities; or explained them on the basis of the
TABLE

RESULTS OF PANCREATIC TESTS IN 10 PATIENTS WITH AMYOTROPHIC LATERAL SCLEROSIS

\begin{tabular}{lcc}
\hline & $\begin{array}{c}\text { Patients } \\
\text { tested } \\
(\text { no. })\end{array}$ & $\begin{array}{c}\text { Abnormal } \\
\text { studies }\end{array}$ \\
\hline Serum amylase & 9 & 0 \\
Urine amylase & 9 & 0 \\
Serum lipase & 9 & 0 \\
Leucine aminopeptidase & 9 & 0 \\
Secretin & 4 & 0 \\
I'1s triolein & 8 & 0 \\
Faecal fat & 9 & 0 \\
Pancreatic scan & 6 & 0 \\
Upper GI x-ray series & 8 & $3^{*}$ \\
Small bowel x-ray series & 8 & $2^{*}$ \\
Glucose tolerance test & 10 & 8 \\
IV tolbutamide test & 8 & $3^{*}$ \\
\hline
\end{tabular}

*See text.

patients' age, previous steroid treatment, or loss of muscle mass.

Steinke and Tyler (1964) performed oral and intravenous glucose tolerance tests and intravenous tolbutamide tests on 13 ALS patients and showed an abnormal glucose utilization in 11 . Two patients had

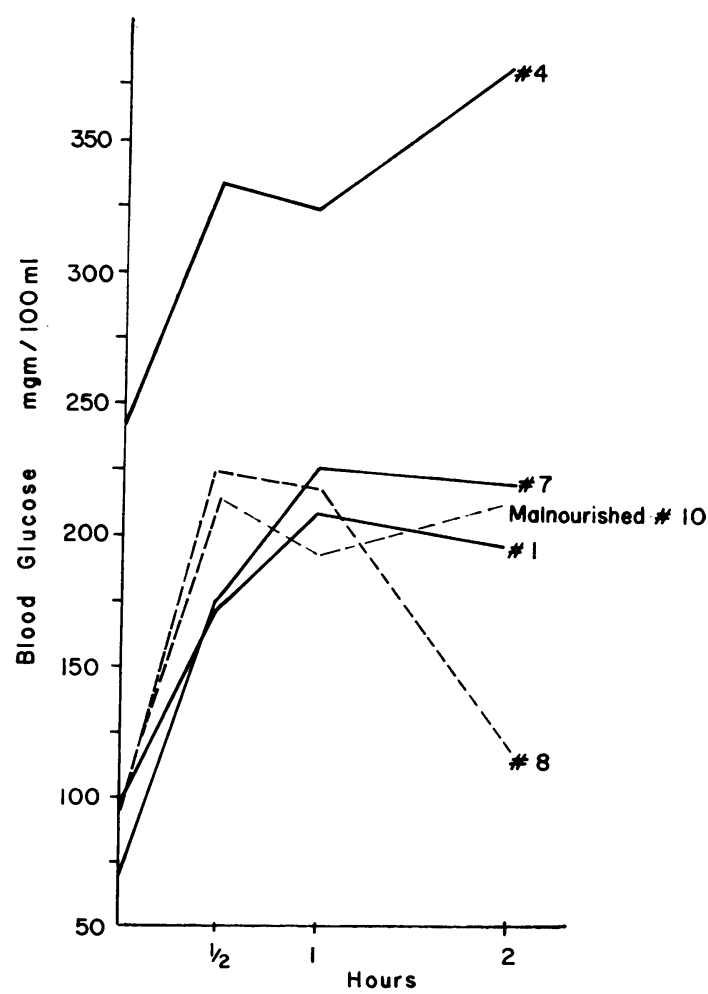

FIG. 1. Glucose tolerance curves. $-=$ diabetic tolbutamide test. - - = tolbutamide test not done. 


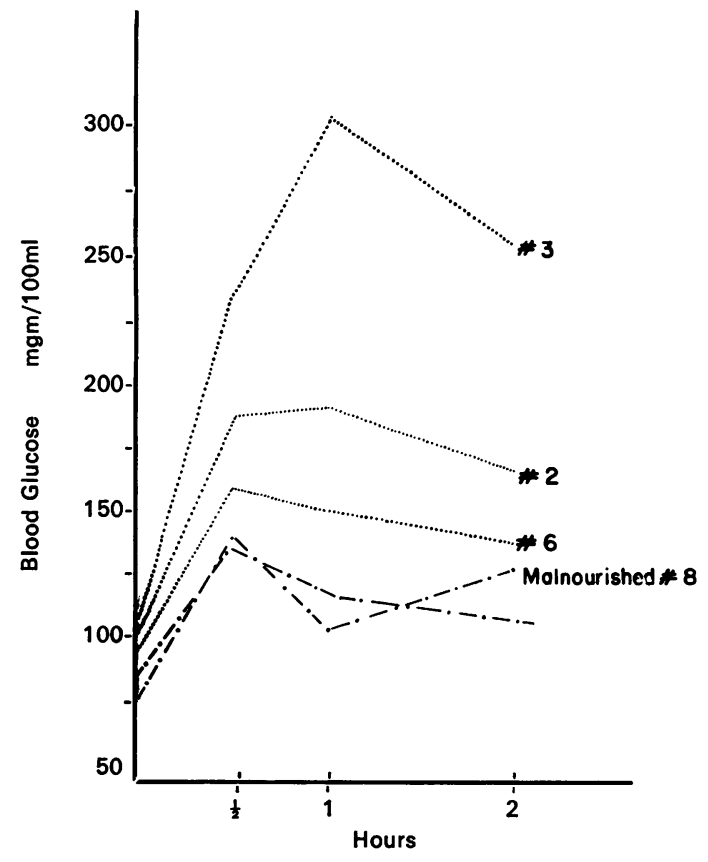

FIG. 2. Glucose tolerance curves. . . . = inconclusive tolbutamide test. $-\cdot-\cdot=$ normal tolbutamide test.

previously known diabetes mellitus, one had a borderline curve, seven had a diabetic curve, and two had normal curves (one patient had only an intravenous glucose tolerance test). They discussed the relationship of physical inactivity, starvation, and age to abnormal glucose tolerance but even when they had excluded the three bedridden patients-all of whom had diabetic curves - the incidence was still greater than in the general population.

Ionăşescu and I.uca (1964) found a decrease in the glucose tolerance in nine out of 15 patients with ALS. They suggested that the abnormal glucose utilization could be explained by the age of the patients, previous treatment with cortisone, and loss of muscle mass. They also found normal arterial-venous differences in glucose during fasting states and during glucose tolerance. Therefore, they concluded, the carbohydrate metabolism in muscle was normal.

Quick and Greer (1967) found four out of five ALS patients had abnormal tolbutamide tests, but all five patients had normal glucose tolerance tests. They were the first to find abnormalities in the exocrine function of the pancreas. More recently Brown and Kater (1969), found normal intravenous glucose tolerance and intravenous tolbutamide tests in seven and five ALS patients respectively.

We believe age, diminished physical activity, decreased muscle mass, and malnutrition can ade- quately explain the abnormal glucose tolerance in six of our patients (all except cases 3 and 4).

Brandt (1960) has shown that in a selected aged population there is a 30 to $60 \%$ prevalence of decreased carbohydrate tolerance. His findings also suggested a relationship between decreased glucose tolerance and physical inactivity. Subsequently, Buhr (1963) has demonstrated such a relationship.

Zierler and Lilienthal (1953) have shown that when muscle mass decreases, the glucose which would have normally entered the muscle accumulates in the blood. The decrease in muscle mass in ALS is well established and may be significant early in the course of the disease.

In the chronic diseases, such as ALS, malnutrition has to be excluded. The picture of 'starvation diabetics' is well known. Only one of our patients could possibly have had this. Malabsorption did not appear to be a factor-even though we did not perform a complete battery of absorption tests. However the glucose tolerance curve would be flat in malabsorption rather than of diabetic type.

None of the eight patients with abnormal glucose tolerance tests had a history of abnormal glucose metabolism before the development of ALS. Three of the eight patients with abnormal glucose tolerance tests were specifically tested before the onset of ALS and had normal glucose metabolism.

We have followed case 4 with great interest, but in spite of good diabetic control her disease has steadily progressed. The patients with abnormal glucose tolerance tests might be labelled as diabetic amyotrophy by some physicians, but we believe their? clinical course precludes that diagnosis. In addition, the clinical picture of an upper motor neurone involvement in all of our patients is against such a diagnosis. One of the deceased patients had a diabetic tolerance curve, and the necropsy was typical for ALS. Finally, we believe that the magnitude of the diabetes is not proportional to the severity of the neurological disease.

More cases of ALS will have to be examined in more detail during earlier stages of the disease before a definite statement concerning the relationship to the endocrine function of the pancreas can be made. However, since this remains the only fairly consistent abnormality it deserves further evaluation.

Glucose tolerance, an endocrine function of the pancreas, is less specific for pancreatic disease than tests of exocrine function. Our complete battery of exocrine tests was normal.

Quick and Greer (1967) were the first to study the exocrine function of the pancreas in ALS. They found five out of five patients had abnormal leucine aminopeptidase levels, four out of four had abnormal secretin tests, and four out of five had 
abnormal $\mathrm{I}^{131}$ triolein and tolbutamide tests. Brown and Kater (1969) refuted these findings by testing the pancreatic function in 10 ALS patients. The secretin tests were normal in all but two patients who had low bicarbonate levels which they thought were secondary to poor collection and a poor batch of secretin. Stool fat content was normal in the eight patients tested. As mentioned previously intravenous glucose tolerance tests were normal in seven patients and intravenous tolbutamide in five patients.

Brown and Kater (1969) suggested that the abnormal pancreatic function may be on the basis of malnutrition secondary to bulbar palsy. Their patients did not have severe bulbar involvement, whereas these of Quick and Greer (1967) did. Seven of our patients had bulbar involvement and four had severe involvement. This would tend to exclude malnutrition as the sole explanation.

In summary, the only abnormalities we found were those of endocrine function. We believe the abnormalities in glucose utilization might be explained on the basis of age, malnutrition, diminished physical activity, and decreased muscle mass. We were unable to confirm the previously reported abnormalities of the exocrine system.

\section{REFERENCES}

Ask-Upmark, E. (1950). Amyotrophic lateral sclerosis observed in 5 persons after gastric resection. Gastroenterology, 15, 257-259.

Barris, R. W. (1953). Pancreatic adenoma (hyperinsulinism) associated with neuromuscular disorders. Ann. intern. Med., 38, 124-129.
Beierwaltes, W. H., Johnson, P. C., and Solari, A. J. (1957). Clinical Use of Radioisotopes. Saunders: Philadelphia.

Brandt, R. L. (1960). Decreased carbohydrate tolerance in elderly patients. Geriatrics, 15, 315-325.

Brown, J. C., and Kater, R. M. H. (1969). Pancreatic function in patients with amyotrophic lateral sclerosis. Neurology (Minneap.), 19, 185-189.

Bühr, P. A. (1963). Über den Einfluss länger dauernder körperlicher inaktivität auf die Blutzucherkurve nach oraler glukosebelastung. Helvetica medica acta, 30, 156-175.

Goldbarg, J. A., and Rutenburg, A. M. (1958). The colorimetric determination of leucine aminopeptidase in urine and serum of normal subjects and patients with cancer and other diseases. Cancer (Philad.), 11, 283-291.

Henry, R. J., Sobel, C., Berkman, S. (1957). On the determination of 'Pancreatitis lipase' in serum. Clin. Chem., 3, 77-89.

Ionăsescu, V., and Luca, N. (1964). Studits on carbohydrate metabolism in amyotrophic lateral sclerosis and hereditary proximal spinal muscular atrophy. Acta neurol. scand., 40, 47-57.

Quick, D. T., and Greer, M. (1967). Pancreatic dysfunction in patients with amyotrophic lateral sclerosis. Neurology (Minneap.), 17, 112-116.

Rice, E. W. (1959). Improved spectrophotometric determination of amylase with a new stable starch substrate solution. Clin. Chem., 5, 592-596.

Steinke, J., and Tyler, H. R. (1964). The association of amyotrophic lateral sclerosis (motor neuron disease) and carbohydrate intolerance, a clinical study. Metabolism, 13, 1376-1381.

Stiel, J. N. (1967). The incidence of gastrectomy and gastric secretion in motor neuron disease. Aust. Ann. Med., 16, 176-177.

van de Kamer, J. H., ten Bokkel Huinink, H., and Weyers, H. A. (1949). Rapid method for the determinations of fat in feces. J. biol. Chem., 177, 347-355.

Zierler, K. L., and Lilienthal, J. L., Jr. (1953). The myopathies: Including their appearance in constitutional disease. Amer. J. Med., 15, 829-844. 